\title{
Ein operativ geheilter Milzabsceß nach Typhus abdominalis.
}

Von Dr. Paul Esau, zurzeit Volontär-Assistent der Mediz.inischen Universitätsklinik in Straßburg.

Wenn ich diesen schon in einer Dissertation verwerteten Fall noclunals veröffentliche. so geschielit es ans dem Grunde. weil ich aus der Mitteilung Federmanns (Deutsche medizinische Wochensclirift 1905, No. 15) ersehe, diß er in der Fülle cler Ijiteratur verscliwinden ist, da bekanntermaßen niemand Dissertationen liest. Dil es aber ein ganz instruktiver Fall war und Milzabscesse auch niclit z.u clen alltäglichen. Dingeu gerechnet werden dürfen, hole ich ihn ınit der gütigen Frlaubnis von Herm Prof. Moritz (Gießen) wieder ans Tageslicht.

Friederike S., Arbeiterfran ron 38 Jalıren, wurde am 26. Augnst 1902 in die Medixinische Klinik zu Greifswald anfgenommen.

Anamnese: Familiär niclits von Belang, Patientin will frölier nie krank gewesen sein. Yor vier Wor:hen erkrankte sic mit Kopfschmer\%en. zul denen sich später noch Durchfälle gesellten. Vor 14 'Tagen (12. Angust) mul(3te Patientin sich ins Bett legen, da die Durchfälle hänfiger (bis achtmal täglich) winden und sie sich sehr scluwach füllte. Jetzt klagt Paticntin ïber Durchfälle. Anch will sie seit der Frkrankung etwas selıwerhörig geworden seiı.

Status praesens: Patientin ist von mittlerer Statur, Muskulatur und Fettpolster mällig entwickelt. Anf der Haut voul Brust und Bancl findet sich eine große Zahl von auffallenden Roseojen. Atnung rulig und gleichmäßig. Frequen 22 p. m. An den Isungen nichts Besonderes. Herædämpfung nicht verbreitert. Der Spitzenstoß ist im fünften Interkostalranm inuerlalb der Mamillarlinie fïhlbar, an der Hersspitze ist ein deutliches systolisches Geränssch hörbar. Temperatur $38,7^{\circ}$. Puls weich, 108 p. m. Leib auf retricben; Bauchdecken sehr dünı und schlaff, Peristaltik deutlich sichtbar. Die Leber erselieint etwas gesenlit. Nilz clentlich palpabel. Stulll dünnfliissig, von grïnlich-bräınlicher Beschaffenheit; der Urin geht gleiclizeitig nuit dem Stıhl ab.

30. Angust. lin katheterisierten Urin deutlicher Gehalt von Eiweil3; Diazoreaktion +

4. September. Besonders rechts starke diffuse Bronchitis, kein Husten. Roseolen verschwunden.

כ. September. Ueber beiden Inngren großblasiges Rasseln.

9. September. Oedem des rechten Fußjes und Unterschenkels ohne Schmeryhaftigkeit, verschwindet Tags darauf.

14. Septennber. Klagen über stärkere Schmerzen im rechten Bein, Anschwellung des recliten Obersehenkels.

16. September. IIintel inten reclits, unterhalb des achten Brustwirbels, Dämpfung und Knisterrassch, ebenso hinten links, unterlaalb des achten Bristwirbels, Dämpfung und Bronchialatmen; keiuo Rasselgeräusche. Vercinzelt Giemen iiber der ganzen Lunge. Patientin klagt ïber Schmerzen in Rüicken.

20. September. Ininks hinten unten eine handbreite, absolute Dämpfung und abgescliwächtes Bronchialatmon; keine Rasselgeräusche. In der ganzen Herıgegend tympanitischer Klang, Hel\%. töne nicht fern klingend.

23. September. Ueber der rechten Lunge ist dic sclion bestehende Dämpfung grölber geworden; reichliches Knacken.

26. September. Abends Temperatursteigerung auf $40,7^{\circ}$. 
27. September. Morgentemperatur $39,2^{\circ}$. Patientin schwitzte stark. Stuhl diarrhoisch, Milz palpapel.

29. September. Rechts hinten unten Rasseln, links hinten unten, vom sechsten Brustwirbel an abwärts, absolute Dämpfung, Stimmfremitus aufgehoben, abgeschwächtes Bronchialatmen, kein Rasseln. Links vorn tympanitischer Schall, desgleichen über dem Herzen; Herz nicht nach rechts verdrängt. In der vorderen linken Axillarlinie bis zur Höhe der Mamilla Dämpfung; Herztöne leise.

30. September. Absolute Herzdämpfung rechts durch den linken Sternalrand, oben durch die vierte Rippe gebildet, Herzspitzenstoß nicht fühlbar. Im fünften Interkostalraum deutliches systolisches Geräusch; über der Mitte des Herzens lautes pericardiales Reiben, das nach der Basis zu schwächer wird. Am Herzrand mit der Atmung synchrones, schwaches Reiben hörbar, das auch mit aufgelegter Hand fühlbar ist.

3. Oktober. Starker Schweiß in der letzten Nacht, Temperatur 36,10. Auch hinten vereinzeltes Reiben hörbar.

9. Oktober. Grenze der absoluten Dämpfung hinten links um einen Wirbelkörper tiefer. Seit zwei Tagen Herpes labialis. Klagen über Stiche in der linken Seite. Reiben nirgends hörbar.

13. Oktober. Schmerzen unter dem linken Rippenbogen wurden bei tiefem Atemholen heftiger; ein Tumor ist fühlbar, welcher bei Palpation ziemlich schmerzhaft zu sein scheint. Dämpfung über den Lungen geht auf der ganzen linken Seite bis zur Mamillarlinie in Höhe der Mamilla. Zweiter Aortenton verstärkt.

15. Oktober. Klagen über anfallsweise auftretende Schmerzen in der linken Seite. Der Tumor (für Milz angesprochene Resistenz) ist nicht zu fühlen. Bauchdecken straff gespannt. Exsudat links unverändert; die Probepunktion am folgenden Tage ergibt klare, etwas blutig gefärbte Flüssigkeit.

17. Oktober. Starke Schmerzen unter dem linken Rippenbogen; wegen der gespanten Bauchdecken ist jedoch nichts zu fühlen.

18. Oktober. Schall über der rechten Spitze hinten verkürzt Lungengrenzen rechts hinten unten nicht gut verschieblich. Links hinten vom fünften Brustwirbel an Dämpfung, die vom sechsten nach unten zu absolut ist. Dieselbe reicht horizontal nach vorn und geht in Höhe der vierten Rippe in die Herzdämpfung über Stimmfremitus über der Dampfung aufgehoben. Ueber der rechten Spitze Vesikuläratmen; nächtliche Schweiße. Bei Betastung spannt sich die Bauchdecke zwischen linkem Rippenbogen und Darmbeinkamm reflektorisch; nnter dem straffen Musculus rectus ist noch eine Resistenz fühlbar. Aus dem linken Hypochondrium tritt ein Tumorhervor, der in seinen Konturen dem vorderen Milzpole entspricht; er hat eine Breite von etwa $11 \mathrm{~cm}$ und überragt den Rippenrand um $6 \mathrm{~cm}$. Nach hinten und oben ist die Milz nicht abzugrenzen

21. Oktober. Milztumor unverändert, zeitweise Schmerzen.

28. Oktober. Punktion des Tumors im linken Hypochondrium; es wurde sanguinolenter Eiter entleert, aus dem sich Typhusbacillen in Reinkultur züchten ließen.

30. Oktober. Der milzförmige Tumor im linken Hypochondrium ist seit dem 18. Oktober besonders in die Breite gewachsen. Er ist am Rippenbogen $14 \mathrm{~cm}$ breit, überragt i hn u $9 \mathrm{~cm}$. Linke Seite etwas vorgewölbt. In halbrechter Seitenlage etwas Dämpfung in den abhängigen Teilen links. Tumor bei der Respiration nicht verschieblich. Pleuraexsudat erheblich zurückgegangen, es überragt die rechte untere Lungengrenze um etwa $1 \frac{1}{2}$ Wirbelkörper.

31. Oktober. Patientin wird auf die chirurgische Station mit der Diagnose "Typhöser Milzabsceß“ verlegt. In Chloroformnarkose wird folgende Operation von Herrn Prof. Bier sofort ausceführt. Etwa $7 \mathrm{~cm}$ langer Schnitt dicht unterhalb des linken Rippenbogens, demselben parallel. Nach Durchschneidung der Bauchdecken kommt man auf harte, fibröse Schwarten. Probepunktion zunächst negativ; dann wird die Spritze schräg nach oben und hinten eingestoßen und nun wird gelber Eiter entleert. Inzision in derselben Richtung. Etwa $200 \mathrm{ccm}$ Eiter, der mit Fetzen von Milzgewebe vermischt war, werden zutage gefördert. Typhus bacillen darin nachgewiesen. Erweiterung der Oeffnung mit dem Dilatator; Einlegen eines starken Drains; Verband. In den nächsten Tagen mäßige Sekretion, einige nekrotische Milzfetzen stoßen sich noch ab. Temperatur nach der Operation sofort zur normalen abgesunken.

13. November. Es entleeren sich noch nekrotische Fetzen; di Eiterhöhle hat sich stark verkleinert. Patientin hat sich gut erholt und an Gewicht erheblich zugenommen.

13. Dezember. Patientin wird als geheilt entlassen.
Man sieht verhältnismäBig selten, aber doch nach einer ganzel: Reihe von Krankheiten, besonders infektiösen, das Auftreten vo Milzabscessen, z. B. bei Polyarthritis rheumatica, Febris recurrens Ruhr, Variola vera, Malaria, nach Endocarditiden, auf tuberculöser ?? Basis, bei puerperalen und Wundinfektionskrankheiten. Außerden liegen eine Anzahl von Beobachtungen vor, in denen man sich dif Art ihrer Entstehung nicht recht vorstellen kann; dahin gehöre: Milzabscesse nach Traumen, Ueberanstrengungen, Alkoholmißbrauch. Erkältung, Leukämie, Scorbut und Purpura. Endlich kommen Milzabscesse vor, bei denen eine Ursache nicht festzustellen ist.

Milzabscesse nach Typhus sind bis jetzt in 20 Fällen beobachtet worden; 13 Patienten sind ohne Operation gestorben, dreimal wurde dabei Typhusbacillen im Absceßeiter post mortem nachgewiesen. Fünf Patienten wurden mit Erfolg operiert, und bei ihnen fanden sicl zweimal Typhusbacillen, zweimal war der Eiter steril, einmal wurd eine bakteriologische Untersuchung nicht gemacht. Bei einem sechsten Patienten, der nach der Operation starb, fanden sich Streptococcen im Absceßeiter. Auch Kruse (in Flügge, Mikroorganismen, Bd. 2) gibt an, einen Fall von Milzabsceß mit Typhusbacillen gesehen zu haben; doch scheint dieser Fall nicht ausführlich veröffentlicht worden zu sein.

Für operierte Patienten ergibt sich also ein vorzügliches Re sultat, und dieses weist den Arzt auf die einzig erfolgversprechende Art der Behandlung hin. Ueber den Modus der Entstehung von Milzabscessen braucht man sich heutzutage keinen großen Spekulationen mehr hinzugeben, da in 85 bis $90 \%$ aller Typhusfälle - manche Statistiken weisen noch höhere Ziffern auf - Eberthsche Bacillen im Blute kreisen. Es wäre vielmehr nicht wunderbar, wenn sich beim Typhus, wie bei anderen septischen Prozessen, viel öfter Abscesse bildeten; immerhin sind ja eitrige Prozesse im Gehirn, den Lungen, der Leber, den Nieren und der Blase, eitrige Affektionen der Pleura, des Pericards, Phlegmonen der Bauchdecken und de: Extremitäten, ulceröse Prozesse an der Trachea schließlich Entzündungen der verschiedensten Knochen und Gelenke im Verlaufe des Typhus bekannt. Lnd ziemlich oft sind aus den entzündlichen Herden Typhusbacillen in Reinkultur gewonnen worden. deren eitererregende Wirkung Roux schon im Jahre 1888 experimentell nachgewiesen hat.

Die Diagnose eines Milzabscesses ist außerordentlich schwer: Auf eines der auffallendsten Zeichen wies ich schon 1903 hin: die ausbleibende Rekonvaleszenz nach dem Abklingen der primären Krankheit; ein solcher Patient verfällt immer mehr und bekommt ein außergewöhnlich kachektiscties Aussehen. Dazı gesellen sich Fröste, Schweiße, hektisches Fieber. Federmann mißt einer gleichzeitig bestehenden linkseitigen Pleuritis großen diagnostischen Wert bei; es können aber auch gerade Pleuritiden den eigentlichen Krankheitssitz verschleiern, weniger allerdings dann, wenn die Affektion der Pleura im Tergleich zu den schweren Allgemeinerscheinungen gering ist. Dem Sitz nach kommen differential-diagnostisch Empyeme und subphrenischer Absceb vor allem in Betracht. Für die Diagnose Won Wichtigkeit sind ferner die Schwellung und besonders das rapide Wachstum der Milz, die linkseitige Spannung der Bauchdecken und endlich der Umstand, daß der Kranke seine linke Seite, besonders bei der Atmung, aufs sorgsamste schont. Oefters hat man analog den Schmerzen in der rechten Schulter bei Lebererkrankungen, bei Milzabscessen, in der linken Schulter Schmerzen beobachtet.

Die Zuhilfenahme der Blutkörperchenzählung scheint sowohl für die Diagnose als auch zur Bestimmung des geeignetsten Operation termins großen Wert zu besitzen; doch ist sie kein unfehlbares Hilfsmittel.

Auf die Prognose und die Behandlung brauche ich nicht weiter einzugehen; die erstere ist bei rechtzeitiger Operation gut, die letztere ist eine chirurgische und wird sich den einzelnen Fülle anpassen müssen, wenn es sich um die Wahl des Weges zur Eröff nung des Abscesses handelt. Eine Eröffnung des Pleuraraume sollte immerhin vermieden werden.

Meistens erholen sich die Patienten nach der Operation sehr rasch, und man braucht nur auf eine Allgemeinbehandlung roborierender Art zu achten, da solche Kranke sich gewöhnlich in einem außerordentlich heruntergekommenen Zustande befinden, un der Appetit wie nach Ablauf eines gewöhnlichen Typhus sich meist rasch und kräftig einstellt. 